\title{
Impact of Educational Guideline on Nursing Students' Knowledge, Beliefs and Attitudes toward Oocyte Cryopreservation
}

\author{
Magda Fawzy Hasab Allah ${ }^{1}$, Safaa Ali Abdelnaem ${ }^{2}$ \&Ola Nabil Abuzaid ${ }^{3}$ \\ 1. Lecturer of Woman Health and Obstetrics Nursing, Faculty of Nursing -Minia University, Egypt. \\ ${ }^{2 .}$ Lecturer of Woman Health and Obstetrics Nursing, Faculty of Nursing -Minia University, Egypt. \\ ${ }^{3 .}$ Lecturer of Community Health Nursing, Faculty of Nursing - Minia University, Egypt.
}

\begin{abstract}
:
Oocyte cryopreservation is an essential means for assisted reproductive technology; it can markedly contribute to advances in infertility treatment and reproductive biology. Aim: To evaluate the impact of educational guideline on nursing students' knowledge, beliefs and attitudes toward oocyte cryopreservation. Research design: quasiexperimental pretest/posttest research design was utilized. Sample: a cluster randomized sample include 125 female students at Faculty of Nursing - Minia University, from the 1 st, $2^{\text {nd }}, 3^{\text {rd }}$ and $4^{\text {th }}$ academic level that include $47,33,22$, 23 respectively. Tools: data were gathered via three tools: the first include socio-demographic data and knowledge assessment tool, the second include beliefs assessment tool, and the third attitudes assessment tool regarding oocyte cryopreservation. Results: $55.2 \%$ of studied students had poor knowledge scores in pretest reduced to $17.6 \%$ of them in posttest with highly statistically significant difference. Furthermore, $60.0 \%$ of them had negative beliefs about egg freezing in pretest vs. $33.6 \%$ in posttest with statistically significant difference. Additionally, only $25.6 \%$ of them had positive attitude about egg freezing in pretest increased to $56.8 \%$ in posttest with statistically significant difference. Conclusion: There were highly statistically significant improvements in students' knowledge, beliefs and attitudes about oocyte cryopreservation after implementation of educational guideline; Recommendation: Conducting health education programs about oocyte cryopreservation on a large sample in non-medical faculties to increase their awareness about it.
\end{abstract}

\section{Keywords: Oocyte Cryopreservation (OC), Nursing Students, Knowledge, Beliefs \& Attitudes.}

\section{Introduction}

Fertility preservation is a new clinical discipline which aimed to maintain the ability to reproduce of young adults and children at risk of infertility. The field is developing rapidly, enhanced by advances in assisted reproductive technologies and cryopreservation methods, in addition to surgical advances. Fertility preservation potential is an important issue for young people at risk of becoming infertile due to clinical conditions, illnesses, or medications. (Rodriguez, et al, 2021)

Egg freezing as a personal choice, also called social egg freezing, is defined as oocyte cryopreservation (OC) for potential future use by women who are choosing to delay childbearing for personal causes, such as career pursuits, progress in education, lack of a partner or financial stability. The first successful human OC and fertilization was reported in 1986 by $\mathrm{C}$ Chen, during which he achieved a successful twin pregnancy. Cryopreservation for medical causes, such as cancer, is already considered as an established method, but it is a fairly new conception for social reasons. (Nasab, et al., 2020)

Fertility preservation gave hope to many people around the world to have children. In the last 30 years, all over the world, statistics showed that almost three million infants have been delivered using ART (European Society of Human Embryology \& Reproduction, 2016).

During the previous few decades, ovarian reserve could be estimated. The newest innovation in assisted reproduction is oocyte cryopreservation, generally known as egg freezing. Fertility-threatening medical conditions or age-related fertility drop can now freeze their eggs, potentially preserving their ability to conceive a genetically related child. Calling egg freezing "one of the most significant latest innovations in assisted reproductive technology". A report in 2019 showing the wide global spread of egg freezing was issued by the International Federation of Fertility Societies (IFFS) (Inhorn et al., 2018a \& 2018b)

Fertility "insurance" is common because the decline in woman's fertility in connection with aging is fast and age "has a unique, irreversible, and terribly negative effect on female fertility." (Gordan, 2015). A woman loses about $95 \%$ of her eggs by age 35, and the remaining $5 \%$ deteriorate rapidly. Fertility declines from $86 \%$ at age 20 to $52 \%$ at age 35 , and then to $36 \%$ at age 40 , and to only $5 \%$ at age 45 . This is a biased reality for women who decide to utilize 
the oocyte cryopreservation process after age 30 to ensure against infertility because a single procedure may not extract any viable eggs due to deterioration. (Biswas \& Selvaraj 2018).

An advanced social trend concerning postponing childbearing has been noted in women of reproductive age over the last three decades, in Western countries. This delay is related to different factors regarding lifestyle and societal changes, such as improved educational and professional chances for women, familial care commitments, economic problems and the need for better financial security, absence of a spouse, necessity of creating a stable home environment, improved access to contraception or a feeling of not being "ready" for parenting. As a consequence, age-related infertility can affect these women when they decide to conceive, and fertility maintenance techniques can be suggested to them as a solution. (Crawford, Steiner, 2015; \& Stoop et al., 2014)

Until nowadays, limited choices for preserving fertility in order to postpone childbearing were offered. Although oocyte freezing and successful thawing is now available, it remains vague to what extent females are aware of the availability of this technique, their attitudes towards its use, or the conditions under which this technique may be considered. Women in western communities have autonomy and choice, over virtually every aspect of their lives: marriage, family planning, work and communications. However, the "biological fertility clock" represents one region that still out of their control, and most women desiring to conceive in their late thirties faced a reduced chance of conception, either spontaneous or through in vitro fertilization (Lallemant, et al, 2016)

Oocyte cryopreservation process takes almost 4 to 6 weeks for a single retrieval. The procedure of egg retrieval includes "2-4 weeks of self-administered hormone injections and birth control pills to temporarily turn off natural hormones" and "10-14 days of hormone injections to motivate the ovaries and ripen numerous eggs." The treatments have been reported to cause "nausea, bloating, and discomfort," possibility of "blood clots, organ failure" "hospitalization" and ovarian hyper stimulation syndrome (Nicolette, 2016).

Many were unaware of the process and those who liked the idea mentioned cost as being the chief hindering factor. In low income regions, things like oocyte cryopreservation are seen as a science fiction luxury. Also in a culture where there is still extreme high importance of virginity and an intact hymen until marriage, most oocyte retrievals would have to be done trans-anally or by the use of laparoscope rather than through-vagina. Even then, in most rural areas even the knowledge that an unmarried woman underwent a gynecological procedure may greatly reduce her marriage opportunities. (Abdelwahab \& Samy al., 2017).

Cryopreservation plays an important role in assisted reproduction, as it enables the reproductive cells collected and/or fertilized in one treatment cycle, to be used for fertilization and/or implanted into the female patient's uterus, in a future treatment cycle. This increases the efficiency of infertility treatment and reduces the cost and discomfort to the patients involved (Kim, et al, 2018).

Awareness about elective egg freezing varies widely, even among medical and nursing staff. Therefore, it is important to inform about fertility preservation at early age. Nursing students as a healthcare professional and as care providers for patients must be aware of fertility maintenance options. In addition, they must be capable of referring patients to a reproductive medicine' team for further a fertility preservation counseling (Rafiei, et al., 2020).

It is well-known that nurses have a significant role in the care and education of patients as well as increasing the awareness about oocyte cryopreservation and following the rights of the patients, especially the right to understand their fertility preservation choices. Additionally, beside their counseling and educating role within the society, nurses have more relations with patients and their families if compared with other healthcare givers. Lately, findings from previous studies revealed that from the physicians' viewpoint, it is the nursing responsibility to discuss about fertility preservation options. (Rafiei, et al., 2020).

Based on the above-mentioned, it was noticed the great role played by nursing staff in education and counseling others regarding fertility preservation, so there is a necessity to improve nursing student's awareness about this topic to serve themselves first and serve others.

\section{Significance of the study:}

Infertility affects 8 to 12 percent of reproductive-age spouses all over the world. However, the rates of infertility in some regions are much higher, reaching $30 \%$ in some areas. In Egypt, infertility ratio has been estimated to be $10.4 \%$ between married couples (Ghraib \& Khait, 2017).

In spite of the all benefits of cryopreservation, there are many factors affecting the couples decision to perform this procedure like the awareness and perception of the couples regarding it. The main role of the nurses is to assess the awareness and perceptions of infertile couple regarding cryopreservation to give more explanation or modification for their concepts (Farrag, \& Eltohamy 2020). 
These young populations (nurses) are thinking about their future reproductive choices as they prepare for a demanding professional career following university courses and because their age is suitable for beneficial oocyte cryopreservation (early and mid-20s). In Egypt, there were scattered researches carried out to assess this issue (Tozzo, et al., 2019).

Aim of the study:

To evaluate the impact of educational guideline on nursing students' knowledge, beliefs and attitudes toward oocyte cryopreservation.

\section{Research hypothesis:}

- Total scores of studied students' knowledge, beliefs and attitude about oocyte cryopreservation will be significantly increased after educational program.

- A significant correlation between knowledge, beliefs and attitudes scores might be found.

\section{Subjects and Methods:}

Research design: Quasi-experimental study design (pre and post-test) was used.

Setting: The current study was carried out on female nursing students at the Faculty of Nursing, Minia University.

Sample type: A cluster random sample was utilized.

Sample Size: Total number of undergraduate nursing students (female only) at Faculty of Nursing - Minia University is (1231) female students for academic year 2020-2021, the $1^{\text {st }}, 2^{\text {nd }}, 3^{\text {rd }}$ and $4^{\text {th }}$ academic level include 449, 336, 213, 233 female students respectively. The researchers took a cluster random sample about $10 \%$ from total number of students which are (125) student. The $1^{\text {st }}, 2^{\text {nd }}, 3^{\text {rd }}$ and $4^{\text {th }}$ academic level that include 47, 33, 22, 23 female students respectively based on the following formula:

$$
\begin{aligned}
x & =Z\left({ }^{c} / 100\right)^{2} r(100-r) \\
n & =N x /{ }_{\left((N-1) E^{2}+x\right)} \\
E & =\text { Sqrt }\left[{ }^{(N-n) x} / n(N-1)\right]
\end{aligned}
$$

Where $N$ is the population size, $r$ is the fraction of responses that you are interested in, and $Z(c / 100)$ is the critical value for the confidence level $c$.

The sample was carefully chosen based on the subsequent parameters;

\section{Exclusion parameters:}

1.Male students (as they don't practice obstetric nursing).

2.Female students who refused to participate in the research.

3. Female students who attain any training courses about oocyte cryopreservation.
Study Tools:

Three tools for gathering data were used in the present research:

The $\mathbf{1}^{\text {st }}$ tool (pre/post): an Arabic self-administered assessment tool established by the researchers after reviewing related literature, purpose of the study and the information wanted to be collected. It consisted from two parts.

Part (I): It includes socio-demographic characteristic: such as (name, age, academic level, residence, marital status, economic status, mother's education and telephone no).

Part (II): Knowledge Assessment Tool (pre/post): It was aimed to evaluate student's knowledge regarding oocyte cryopreservation that includes (17) questions MCQ about (concept of oocyte cryopreservation, Indication, criteria of egg freezing process, risks and complication, needed laboratory tests, source of knowledge about oocyte cryopreservation ..........etc). Scoring system: knowledge questions were assumed scores $(2,1$ or zero). It was given to each answer equal (correct, and incorrect) correspondingly. Overall knowledge scores were categorized as: poor $(<50 \%)$, average $(50 \%-\leq 75 \%)$, good (> 75\%).

$2^{\text {nd }}$ Tool: Beliefs assessment tool (pre/post): an Arabic self-administered questionnaire established by the researchers after reviewing related literature. It was aimed to evaluate subject's beliefs regarding oocyte cryopreservation, that includes (9) questions which reflects the student's beliefs regarding oocyte cryopreservation by using a 4-point Likert scale, each item graded from (1) definitely not, (2) probably not, (3) probably, (4) definitely. Total Beliefs scores were categorized as: Negative $(<60 \%)$ and Positive $(\geq$ $60 \%)$.

$3^{\text {rd }}$ Tool: Attitude assessment tool (pre/post): an Arabic self-administered questionnaire established by the researchers after reviewing related literature. It was aimed to evaluate subject's attitude toward oocyte cryopreservation, that contains (9) questions which reflects the student's attitude toward oocyte cryopreservation by using a 2 point Likert scale, each positive statement from (2) Agree, (1) Disagree, Total attitude scores were classified as: Negative attitude $(<60 \%)$ and Positive attitude $(\geq 60 \%)$.

Validity: The sheet was reviewed by five-panel experts from community health \& obstetrics and gynecological department nursing professors who revised the tools for accuracy, relevance, inclusiveness, understanding, applicability and simplicity.

Reliability: The test by Alpha Cronbach was conducted to evaluate the steadiness of the instruments' internal consistency. Knowledge sheet was 0.870 . It was 0.852 for the assessment of lifestyle 
scale, hence the sheets were found to be highly reliable.

\section{Pilot study:}

It was carried out on $10 \%$ of the overall sample examined (12 student). It was carried out to assess the applicability and clarity of the instruments, evaluate the feasibility of fieldwork, and identify any potential difficulties that the investigator could encounter and impede the collection of data. Important improvements have been made based on the findings of the pilot study, such as (removing certain questions and adding others) to endorse their content or for clearer and more specific purposes. In the basic sample, the trial sample was involved.

\section{Procedure:}

A written approval letter was officially obtained from the Dean, Vice dean of research and post graduate and the head of the department of Obstetrics and Gynecology at Faculty of Nursing Minia University describing the purpose of the current research, Also agreement of ethical committee was taken before conduct this research. The present study was performed in three phases: assessment phase (pretest), implementation phase (carrying out education program), and evaluation phase (immediately posttest).

\section{Assessment phase (pre-test)}

The researchers conducting the first meeting with each educational grade on their free time and briefly discussed the essence and the intent of the study. They have been told that their contribution and their right to discontinuation at any time are optional. Oral approval from all students was achieved.

After having the agreement from the female students to share in the present study, the researchers gave outline and illustration about the evaluation tools to each sample in each academic level. Then, each student received self-administered questionnaire to evaluate the data concerning to socio-demographic characteristics, knowledge, beliefs and attitude. The students had about 15-20 minutes to end the questionnaire.

\section{Implementation phase (carrying out education program)}

After evaluating the students' knowledge, beliefs and attitude toward oocyte cryopreservation by pretest questions sheet. The whole students (125) were classified into small groups (4 groups) according to their academic level. Four sessions were conducted to implement the educational program, one session for each group, each session took about one hour in their free time.

Sessions were given to the students in the form of symposium and group discussion by using audiovisual aids (PowerPoint and videos), it ensure enhancing female student's knowledge, beliefs and attitude. At the finish of the symposium, extra 15 minutes were given for an open discussion about the topic and feedback was attained to confirm that the students acquired the maximum benefits.

Leaflets include information about oocyte cryopreservation were given to female students at the finish of symposium.

\section{Evaluation phase (post-test):}

The influence of the educational sessions on improving knowledge, beliefs and attitudes of students towards oocyte cryopreservation was achieved through a pre- and post-test comparison that was carried out immediately after educational session. The data was collected between the beginning of March 2021 and the end of May 2021 over a threemonth period.

\section{Ethical Concern:}

All formal permits were obtained from convenient authorities to perform the report. The relevance and purpose of this study were discussed with the participants. Both participants were told that their contribution was voluntary and their right to discontinue at any time, that data confidentiality was achieved, and that the data collected was only used for the purpose of the present study.

\section{Statistical analysis}

The data obtained was tabulated using the SPSS version and statistically analyzed (20). When p-value 0.05 , and high significance when p-value 0.001 and no statistically significant difference when $\mathrm{p}$-value > 0.05 is considered, statistical significance difference was considered. Statistical tests were used, including an independent t-test and a Chi-square test. 


\section{Results:}

Table (1): Distribution of studied students' according to their socio-demographic data $(n=125)$.

\begin{tabular}{|c|c|c|}
\hline Socio-demographic data of students & No. & $\%$ \\
\hline \multicolumn{3}{|l|}{ Age/year } \\
\hline $18-<20$ & 76 & 60.8 \\
\hline $20-<22$ & 40 & 32.0 \\
\hline$\geq 22$ & 9 & 7.2 \\
\hline Mean \pm SD & \multicolumn{2}{|c|}{$19.35 \pm 1.2$ years } \\
\hline \multicolumn{3}{|l|}{ Academic level } \\
\hline $1^{\text {st }}$ yrs. & 47 & 37.6 \\
\hline $2^{\text {nd }} y r s$. & 33 & 26.4 \\
\hline $3^{\text {rd }}$ yrs. & 22 & 17.6 \\
\hline $4^{\text {th }} \mathrm{yrs}$. & 23 & 18.4 \\
\hline \multicolumn{3}{|l|}{ Residence } \\
\hline Urban & 33 & 26.4 \\
\hline Rural & 92 & 73.6 \\
\hline \multicolumn{3}{|l|}{ Socio-economic level } \\
\hline Low & 20 & 16.0 \\
\hline Moderate & 103 & 82.4 \\
\hline High & 2 & 1.6 \\
\hline \multicolumn{3}{|l|}{ Marital Status } \\
\hline Single & 115 & 92.0 \\
\hline Married & 10 & 8.0 \\
\hline \multicolumn{3}{|l|}{ Religious } \\
\hline Muslim & 98 & 78.4 \\
\hline Christian & 27 & 21.6 \\
\hline \multicolumn{3}{|l|}{ Mothers' education } \\
\hline Illiterate & 43 & 34.4 \\
\hline Primary or Preparatory & 34 & 27.2 \\
\hline Secondary & 33 & 26.4 \\
\hline University & 15 & 12.0 \\
\hline
\end{tabular}

Table (2): Distribution of studied students' according to their answers about oocyte cryopreservation $(\mathbf{n}=\mathbf{1 2 5})$.

\section{Knowledge about oocyte cryopreservation}

\begin{tabular}{|l|c|c|c|c|} 
& Correct & Incorrect & Correct & Incorrect \\
\hline 1. Meaning of oocyte cryopreservation & 20.0 & 80.0 & 52.0 & 48.0 \\
\hline 2. Indications for oocyte cryopreservation: & 24.8 & 75.2 & 76.0 & 24.0 \\
\hline $\begin{array}{l}\text { 3. There is a significant decrease in a woman's fertility with } \\
\text { advanced age. }\end{array}$ & 50.4 & 49.6 & 92.0 & 8.0 \\
\hline 4. Oocyte cryopreservation can prolong a woman's fertility. & 36.8 & 63.2 & 72.0 & 28.0 \\
\hline $\begin{array}{l}\text { 5. At what age period there is a marked decrease in woman's } \\
\text { fertility? }\end{array}$ & 10.4 & 89.6 & 21.6 & 78.4 \\
\hline $\begin{array}{l}\text { 6. How many eggs should be stored to achieve a pregnancy later } \\
\text { on? }\end{array}$ & 16.8 & 83.2 & 61.6 & 38.4 \\
\hline 7. At what age is it effective for a woman to freeze her eggs? & 10.4 & 89.6 & 62.4 & 37.6 \\
\hline 8. Barriers of oocyte cryopreservation & 2.4 & 97.6 & 15.2 & 84.8 \\
\hline
\end{tabular}




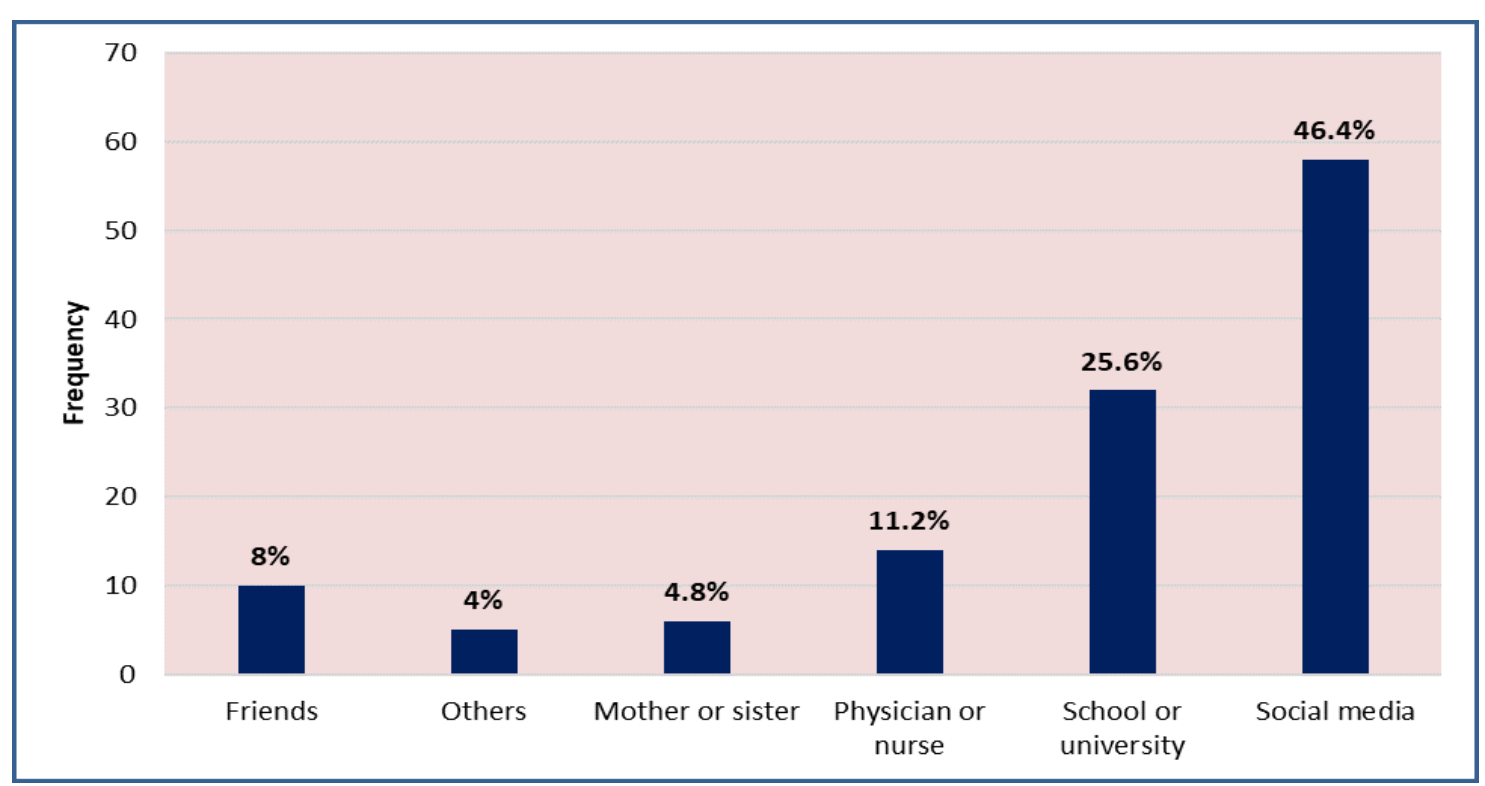

Figure (1): Sources of information about oocyte cryopreservation

Table (3): Distribution of studied students' according to their beliefs about oocyte cryopreservation $(\mathbf{n}=125)$.

\begin{tabular}{|c|c|c|c|c|c|c|c|c|}
\hline \multirow{3}{*}{ Beliefs about oocyte cryopreservation } & \multicolumn{4}{|c|}{ Pretest } & \multicolumn{4}{|c|}{ Posttest } \\
\hline & 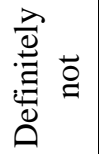 & $\begin{array}{l}\text { ㄱ. } \\
\stackrel{\tilde{\Xi}}{0} \\
\stackrel{0}{0} \\
\stackrel{0}{0}\end{array}$ & $\begin{array}{l}\lambda \\
\overrightarrow{0} \\
00 \\
0 \\
0\end{array}$ & 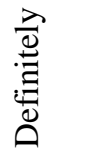 & 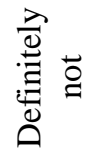 & 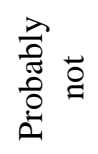 & 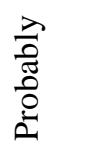 & 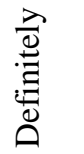 \\
\hline & $\%$ & $\%$ & $\%$ & $\%$ & $\%$ & $\%$ & $\%$ & $\%$ \\
\hline $\begin{array}{l}\text { 1. Woman should freeze her eggs if she } \\
\text { isn't ready to have a child. }\end{array}$ & 28.0 & 19.2 & 38.4 & 14.4 & 5.6 & 9.6 & 24.8 & 60.0 \\
\hline $\begin{array}{l}\text { 2. Woman should freeze her eggs if she had } \\
\text { cancer. }\end{array}$ & 14.4 & 15.2 & 44.8 & 25.6 & 24.0 & 12.0 & 55.2 & 8.8 \\
\hline $\begin{array}{l}\text { 3. Oocyte cryopreservation should be a part } \\
\text { of premarital counselling. }\end{array}$ & 14.4 & 16.8 & 51.2 & 17.6 & 9.6 & 9.6 & 31.2 & 49.6 \\
\hline $\begin{array}{l}\text { 4. The high cost is a barrier for oocyte } \\
\text { cryopreservation. }\end{array}$ & 9.6 & 20.8 & 36.0 & 33.6 & 46.4 & 8.0 & 29.6 & 16.0 \\
\hline $\begin{array}{l}\text { 5. The costs should be covered by national } \\
\text { health care system. }\end{array}$ & 13.6 & 32.0 & 48.0 & 6.4 & 4.0 & 8.0 & 60.0 & 28.0 \\
\hline $\begin{array}{l}\text { 6. I would freeze my eggs if I had not yet } \\
\text { found a suitable partner. }\end{array}$ & 32.0 & 23.2 & 38.4 & 6.4 & 56.0 & 4.0 & 32.8 & 7.2 \\
\hline $\begin{array}{l}\text { 7. Oocyte cryopreservation is forbidden by } \\
\text { your religion. }\end{array}$ & 13.6 & 45.6 & 28.0 & 12.8 & 44.8 & 23.2 & 17.6 & 14.4 \\
\hline $\begin{array}{l}\text { 8. The frozen eggs remain usable for about } \\
10 \text { yrs. }\end{array}$ & 26.4 & 13.6 & 52.0 & 8.0 & 17.6 & 53.6 & 24.0 & 4.8 \\
\hline \multicolumn{9}{|l|}{$\begin{array}{l}\text { 9. After freezing my eggs, I would consider } \\
\text { donating them: }\end{array}$} \\
\hline (a) For medical research. & 42.4 & 24.0 & 16.8 & 16.8 & 30.4 & 21.6 & 38.4 & 9.6 \\
\hline (b) To a friend or family member. & 37.6 & 24.0 & 20.8 & 17.6 & 55.2 & 5.6 & 29.6 & 9.6 \\
\hline (c) To an egg donor program & 37.6 & 20.0 & 24.0 & 18.4 & 28.0 & 56.8 & 11.2 & 4.0 \\
\hline
\end{tabular}


Table (4): Distribution of studied students' according to their attitude toward oocyte cryopreservation $(\mathbf{n}=\mathbf{1 2 5})$.

\begin{tabular}{|c|c|c|c|c|}
\hline \multirow{3}{*}{ Attitude toward oocyte cryopreservation } & \multicolumn{2}{|c|}{ Pretest } & \multicolumn{2}{|c|}{ Posttest } \\
\hline & Agree & Disagree & Agree & Disagree \\
\hline & $\%$ & $\%$ & $\%$ & $\%$ \\
\hline 1. Egg preservation should be encouraged & 55.2 & 44.8 & 72.0 & 28.0 \\
\hline 2. Against any technique that experiments with human beings & 47.2 & 52.8 & 43.2 & 56.8 \\
\hline 3. Egg preservation should be used to exclude genetic disorder & 61.6 & 38.4 & 72.0 & 28.0 \\
\hline 4. Egg preservation is frightening because of its complicates. & 30.4 & 69.6 & 37.6 & 62.4 \\
\hline $\begin{array}{l}\text { 5. A child born with egg preservation will not be healthy or may have } \\
\text { birth defect }\end{array}$ & 43.2 & 56.8 & 32.0 & 68.0 \\
\hline 6. We do not apply egg preservation in Egypt & 44.8 & 55.2 & 28.0 & 72.0 \\
\hline 7. It is very expensive and therefore cannot be feasible to anyone & 64.0 & 36.0 & 24.8 & 75.2 \\
\hline 8. Egg preservation is not very attractive, as the success rate is very low & 46.4 & 53.6 & 34.4 & 65.6 \\
\hline 9. Oocyte freezing may be a possible option for you & 30.4 & 69.6 & 68.0 & 32.0 \\
\hline
\end{tabular}

Table (5): Distribution of studied students according to their total knowledge, beliefs and attitude levels about oocyte cryopreservation $(n=125)$

\begin{tabular}{l|c|c|c|c|c|c}
\multirow{2}{*}{ Total score } & \multicolumn{2}{|c|}{ Pretest } & \multicolumn{2}{c|}{ Posttest } & \multirow{2}{*}{$t$} & \multirow{2}{*}{ P-Value } \\
\cline { 2 - 4 } & No & No & $\%$ & \\
\hline
\end{tabular}

\begin{tabular}{|c|c|c|c|c|c|c|}
\hline \multicolumn{7}{|c|}{ Knowledge about oocyte cryopreservation $(n=125)$. } \\
\hline Poor $<(50 \%)$ & 69 & 55.2 & 22 & 17.6 & \multirow{4}{*}{18.303} & $0.000^{* *}$ \\
\hline Average $(50-75 \%)$ & 49 & 39.2 & 33 & 26.4 & & \\
\hline Good $>(75 \%)$ & 7 & 5.6 & 70 & 56.0 & & \\
\hline Mean \pm SD & \multicolumn{2}{|c|}{$20.21 \pm 10.28$} & \multicolumn{2}{|c|}{$39.75 \pm 8.25$} & & \\
\hline \multicolumn{7}{|c|}{ Beliefs about oocyte cryopreservation $(\mathbf{n}=125)$} \\
\hline Negative $<(60 \%)$ & 75 & 60.0 & 42 & 33.6 & \multirow{3}{*}{1.879} & $0.050^{*}$ \\
\hline Positive $\geq(60 \%)$ & 50 & 40.0 & 83 & 66.4 & & \\
\hline Mean \pm SD & & 5.07 & & 6.47 & & \\
\hline \multicolumn{7}{|c|}{ Attitude toward oocyte cryopreservation $(n=125)$} \\
\hline Negative $<(60 \%)$ & 93 & 74.4 & 53 & 42.4 & \multirow{3}{*}{5.128} & \multirow{3}{*}{$0.000^{* *}$} \\
\hline Positive $\geq(60 \%)$ & 32 & 25.6 & 72 & 56.8 & & \\
\hline Mean \pm SD & & 1.6 & & 2.25 & & \\
\hline
\end{tabular}

Test used: paired sample T test. $\quad *$ Statistically significant at $P-$ value $\leq .05 * * *$ Statistically significant at $P-$ value $\leq .01$

Table (6): Relation between studied student's knowledge about oocyte cryopreservation and their demographic data in pretest and posttest, $(n=125)$.

\begin{tabular}{|c|c|c|c|c|c|c|}
\hline \multirow{3}{*}{ Demographic data of students } & \multicolumn{6}{|c|}{ Knowledge about oocyte cryopreservation } \\
\hline & \multicolumn{3}{|c|}{ Pretest } & \multicolumn{3}{|c|}{ Posttest } \\
\hline & $\begin{array}{c}\text { Poor } \\
(n=69)\end{array}$ & $\begin{array}{c}\text { Average } \\
(n=49)\end{array}$ & $\begin{array}{c}\text { Good } \\
(\mathbf{n}=7)\end{array}$ & $\begin{array}{c}\text { Poor } \\
(\mathrm{n}=\mathbf{2 2})\end{array}$ & $\begin{array}{c}\text { Average } \\
(n=33)\end{array}$ & $\begin{array}{c}\text { Good } \\
(\mathbf{n}=70)\end{array}$ \\
\hline \multicolumn{7}{|l|}{ Age/year } \\
\hline $18-<20$ & 62.3 & 61.2 & 42.9 & 59.1 & 54.5 & 64.3 \\
\hline $20-<22$ & 29.0 & 32.7 & 50.1 & 31.8 & 30.3 & 32.9 \\
\hline$\geq 22$ & 8.7 & 6.1 & 7.0 & 9.1 & 15.2 & 2.9 \\
\hline $\mathrm{X}^{2}(\mathrm{P}-$ value $)$ & \multicolumn{3}{|c|}{$12.781(.030)^{*}$} & \multicolumn{3}{|c|}{$14.849(0.033)^{*}$} \\
\hline \multicolumn{7}{|l|}{ Academic level } \\
\hline 1st yrs. & 47.8 & 26.5 & 14.3 & 18.2 & 42.4 & 41.4 \\
\hline 2nd yrs. & 15.9 & 40.8 & 28.6 & 40.9 & 18.2 & 25.7 \\
\hline 3rd yrs. & 11.6 & 22.4 & 42.9 & 18.2 & 15.2 & 18.6 \\
\hline 4th yrs. & 24.6 & 10.2 & 14.3 & 22.7 & 24.2 & 14.3 \\
\hline $\mathrm{X}^{2}(\mathrm{P}-$ value $)$ & \multicolumn{3}{|c|}{$19.174(.000)^{* * *}$} & \multicolumn{3}{|c|}{$18.921(0.001)^{* * *}$} \\
\hline \multicolumn{7}{|l|}{ Residence } \\
\hline Urban & 24.6 & 24.5 & 57.1 & 31.8 & 39.4 & 18.6 \\
\hline Rural & 75.4 & 75.5 & 42.9 & 68.2 & 60.6 & 81.4 \\
\hline $\mathrm{X}^{2}(\mathrm{P}-$ value $)$ & \multicolumn{3}{|c|}{$3.607(.165)^{\mathrm{NS}}$} & \multicolumn{3}{|c|}{$1.848(.397)$} \\
\hline
\end{tabular}




\begin{tabular}{|c|c|c|c|c|c|c|}
\hline \multirow{3}{*}{ Demographic data of students } & \multicolumn{6}{|c|}{ Knowledge about oocyte cryopreservation } \\
\hline & \multicolumn{3}{|c|}{ Pretest } & \multicolumn{3}{|c|}{ Posttest } \\
\hline & $\begin{array}{c}\text { Poor } \\
(\mathbf{n}=69)\end{array}$ & $\begin{array}{c}\text { Average } \\
(\mathrm{n}=49)\end{array}$ & $\begin{array}{c}\text { Good } \\
(\mathbf{n}=7)\end{array}$ & $\begin{array}{c}\text { Poor } \\
(\mathbf{n}=\mathbf{2 2})\end{array}$ & $\begin{array}{c}\text { Average } \\
(\mathbf{n}=\mathbf{3 3})\end{array}$ & $\begin{array}{c}\text { Good } \\
(\mathrm{n}=70)\end{array}$ \\
\hline \multicolumn{7}{|l|}{ Socio-economic level } \\
\hline Low & 23.2 & 8.2 & 0.0 & 13.6 & 24.2 & 12.9 \\
\hline Moderate & 73.9 & 91.8 & 100 & 81.8 & 75.8 & 85.7 \\
\hline High & 2.9 & 0.0 & 0.0 & 4.5 & 0.0 & 1.4 \\
\hline $\mathrm{X}^{2}(\mathrm{P}-$ value $)$ & \multicolumn{3}{|c|}{$8.248(.083)$} & \multicolumn{3}{|c|}{$6.646(.801)$} \\
\hline \multicolumn{7}{|l|}{ Marital Status } \\
\hline Single & 91.3 & 95.9 & 71.4 & 90.9 & 87.9 & 94.3 \\
\hline Married & 8.7 & 4.1 & 28.6 & 9.1 & 12.1 & 5.7 \\
\hline $\mathrm{X}^{2}(\mathrm{P}-$ value $)$ & \multicolumn{3}{|c|}{$15.092(.002)$} & \multicolumn{3}{|c|}{$13.434(0.003) *$} \\
\hline \multicolumn{7}{|l|}{ Religion } \\
\hline Muslim & 75.4 & 83.7 & 71.4 & 81.8 & 84.8 & 74.3 \\
\hline Christian & 24.6 & 16.3 & 28.6 & 18.2 & 15.2 & 25.7 \\
\hline $\mathrm{X}^{2}(\mathrm{P}-$ value $)$ & \multicolumn{3}{|c|}{$1.382(.501)^{\mathrm{NS}}$} & \multicolumn{3}{|c|}{$1.865(.394)$} \\
\hline \multicolumn{7}{|l|}{ Mothers' education } \\
\hline Illiterate & 36.2 & 28.6 & 42.9 & 45.5 & 30.3 & 31.4 \\
\hline Primary or Preparatory & 27.5 & 29.6 & 14.3 & 22.7 & 33.3 & 25.7 \\
\hline Secondary & 29.0 & 22.4 & 28.6 & 13.6 & 30.3 & 29.0 \\
\hline University & 7.2 & 19.4 & 14.3 & 18.2 & 6.1 & 13.9 \\
\hline $\mathrm{X}^{2}(\mathrm{P}-$ value $)$ & \multicolumn{3}{|c|}{$14.617(.003) * *$} & \multicolumn{3}{|c|}{$15.925(.002)$} \\
\hline
\end{tabular}

Table (7): Relation between studied student's beliefs about oocyte cryopreservation and their demographic data in pretest and posttest, $(\mathrm{n}=125)$.

\begin{tabular}{|c|c|c|c|c|}
\hline \multirow[b]{3}{*}{ Demographic data of students } & \multicolumn{4}{|c|}{ Beliefs about oocyte cryopreservation } \\
\hline & \multicolumn{2}{|c|}{ Pretest } & \multicolumn{2}{|c|}{ Posttest } \\
\hline & $\begin{array}{l}\text { Positive } \\
(\mathbf{n}=\mathbf{5 0})\end{array}$ & $\begin{array}{c}\text { Negative } \\
(n=75)\end{array}$ & $\begin{array}{l}\text { Positive } \\
(\mathbf{n}=\mathbf{8 3})\end{array}$ & $\begin{array}{c}\text { Negative } \\
(\mathrm{n}=42)\end{array}$ \\
\hline \multicolumn{5}{|l|}{ Age/year } \\
\hline $18-<20$ & 56.0 & 64.0 & 57.8 & 66.7 \\
\hline $20-<22$ & 36.0 & 29.3 & 33.7 & 28.6 \\
\hline$\geq 22$ & 8.0 & 6.7 & 8.4 & 4.8 \\
\hline $\mathrm{X}^{2}(\mathrm{P}-$ value $)$ & \multicolumn{2}{|c|}{$11.807(.050)^{* *}$} & \multicolumn{2}{|c|}{$6.408(.403)$} \\
\hline \multicolumn{5}{|l|}{ Academic level } \\
\hline $1^{\text {st }}$ yrs. & 40.0 & 36.0 & 33.7 & 45.2 \\
\hline $2^{\text {nd }} y r s$. & 20.0 & 30.7 & 27.7 & 23.8 \\
\hline $3^{\text {rd }}$ yrs. & 18.0 & 17.3 & 14.5 & 23.8 \\
\hline $4^{\text {th }}$ yrs. & 22.0 & 16.0 & 24.1 & 7.1 \\
\hline $\mathrm{X}^{2}(\mathrm{P}-$ value $)$ & \multicolumn{2}{|c|}{$12.015(.053)^{*}$} & \multicolumn{2}{|c|}{$11.408(.049)^{*}$} \\
\hline \multicolumn{5}{|l|}{ Residence } \\
\hline Urban & 30.0 & 24.0 & 32.5 & 14.3 \\
\hline Rural & 70.0 & 76.0 & 67.5 & 85.7 \\
\hline $\mathrm{X}^{2}(\mathrm{P}-$ value $)$ & \multicolumn{2}{|c|}{$4.556(.294)^{\mathrm{NS}}$} & \multicolumn{2}{|c|}{$3.542(.355)^{\mathrm{NS}}$} \\
\hline \multicolumn{5}{|l|}{ Socio-economic level } \\
\hline Low & 32.0 & 5.3 & 15.7 & 16.7 \\
\hline Moderate & 66.0 & 93.3 & 83.1 & 81.0 \\
\hline High & 2.0 & 1.3 & 1.2 & 2.4 \\
\hline $\mathrm{X}^{2}(\mathrm{P}-$ value $)$ & \multicolumn{2}{|c|}{$16.137(.000)^{* *}$} & \multicolumn{2}{|c|}{$8.677(.454)^{\mathrm{NS}}$} \\
\hline \multicolumn{5}{|l|}{ Marital Status } \\
\hline Single & 94.0 & 90.7 & 90.4 & 95.2 \\
\hline Married & 6.0 & 9.3 & 9.6 & 4.8 \\
\hline $\mathrm{X}^{2}(\mathrm{P}-$ value $)$ & \multicolumn{2}{|c|}{$5.453(.376)^{\mathrm{NS}}$} & \multicolumn{2}{|c|}{$3.817(.051)^{*}$} \\
\hline \multicolumn{5}{|l|}{ Religious } \\
\hline Muslim & 82.0 & 76.0 & 80.7 & 73.8 \\
\hline Cristian & 18.0 & 24.0 & 19.3 & 26.2 \\
\hline $\mathrm{X}^{2}(\mathrm{P}-$ value $)$ & \multicolumn{2}{|c|}{$2.638(.509)^{\mathrm{NS}}$} & \multicolumn{2}{|c|}{$1.032(.858)$} \\
\hline
\end{tabular}




\begin{tabular}{|c|c|c|c|c|}
\hline \multirow[b]{3}{*}{ Demographic data of students } & \multicolumn{4}{|c|}{ Beliefs about oocyte cryopreservation } \\
\hline & \multicolumn{2}{|c|}{ Pretest } & \multicolumn{2}{|c|}{ Posttest } \\
\hline & $\begin{array}{l}\text { Positive } \\
(\mathbf{n}=\mathbf{5 0})\end{array}$ & $\begin{array}{c}\text { Negative } \\
(n=75)\end{array}$ & $\begin{array}{l}\text { Positive } \\
(\mathrm{n}=83)\end{array}$ & $\begin{array}{c}\text { Negative } \\
(\mathrm{n}=42)\end{array}$ \\
\hline \multicolumn{5}{|l|}{ Mothers' education } \\
\hline Illiterate & 34.0 & 33.3 & 27.7 & 45.2 \\
\hline Primary or Preparatory & 32.0 & 24.0 & 30.1 & 21.4 \\
\hline Secondary & 24.0 & 28.0 & 30.1 & 20.4 \\
\hline University & 10.0 & 13.3 & 12.0 & 12.9 \\
\hline $\mathrm{X}^{2}(\mathrm{P}-$ value $)$ & \multicolumn{2}{|c|}{$1.161(.774)^{\mathrm{NS}}$} & \multicolumn{2}{|c|}{$4.041(.774)^{\mathrm{NS}}$} \\
\hline
\end{tabular}

$N S=$ Not statistically significance $*$ Statistically significant at $P-$ value $\leq 0.05 \quad * *$ Statistically significant at $P-$ value $\leq .01$

Table (8): Relation between studied student's attitudes toward oocyte cryopreservation and their demographic data in pretest and posttest, $(n=125)$.

\begin{tabular}{|c|c|c|c|c|}
\hline \multirow{3}{*}{ Demographic data of students } & \multicolumn{4}{|c|}{ Attitudes about oocyte cryopreservation } \\
\hline & \multicolumn{2}{|c|}{\begin{tabular}{l|l} 
Pretest \\
\end{tabular}} & \multicolumn{2}{|c|}{ Posttest } \\
\hline & $\begin{array}{l}\text { Positive } \\
(\mathrm{n}=32)\end{array}$ & $\begin{array}{c}\text { Negative } \\
(n=93)\end{array}$ & $\begin{array}{l}\text { Positive } \\
(\mathrm{n}=72)\end{array}$ & $\begin{array}{c}\text { Negative } \\
(n=53)\end{array}$ \\
\hline \multicolumn{5}{|l|}{ Age/year } \\
\hline $18-<20$ & 56.3 & 62.4 & 63.4 & 56.6 \\
\hline $20-<22$ & 31.3 & 32.3 & 31.0 & 34.0 \\
\hline$\geq 22$ & 12.5 & 5.4 & 5.6 & 9.4 \\
\hline $\mathrm{X}^{2}(\mathrm{P}-$ value $)$ & \multicolumn{2}{|c|}{$13.832(.008)^{* *}$} & \multicolumn{2}{|c|}{$13.367(0.007)$} \\
\hline \multicolumn{5}{|l|}{ Academic level } \\
\hline 1st yrs. & 25.0 & 41.9 & 39.4 & 34.0 \\
\hline 2nd yrs. & 34.4 & 23.7 & 22.5 & 32.1 \\
\hline 3rd yrs. & 21.9 & 16.1 & 16.9 & 18.9 \\
\hline 4th yrs. & 18.8 & 18.3 & 21.1 & 15.1 \\
\hline $\mathrm{X}^{2}(\mathrm{P}-$ value $)$ & \multicolumn{2}{|c|}{$13.302(.002)^{* *}$} & \multicolumn{2}{|c|}{$4.582(.099)^{\mathrm{NS}}$} \\
\hline \multicolumn{5}{|l|}{ Residence } \\
\hline Urban & 40.6 & 21.5 & 25.4 & 26.4 \\
\hline Rural & 59.4 & 78.5 & 74.6 & 73.6 \\
\hline $\mathrm{X}^{2}(\mathrm{P}-$ value $)$ & \multicolumn{2}{|c|}{$4.479(.034)^{*}$} & \multicolumn{2}{|c|}{$0.999(.209)^{\mathrm{NS}}$} \\
\hline \multicolumn{5}{|l|}{ Socio-economic level } \\
\hline Low & 6.3 & 19.4 & 19.7 & 11.3 \\
\hline Moderate & 90.6 & 79.6 & 80.3 & 84.9 \\
\hline High & 3.1 & 1.1 & 0.0 & 3.8 \\
\hline $\mathrm{X}^{2}(\mathrm{P}-$ value $)$ & \multicolumn{2}{|c|}{$8.248(.083)^{\mathrm{NS}}$} & \multicolumn{2}{|c|}{$6.889(.032)^{*}$} \\
\hline \multicolumn{5}{|l|}{ Marital Status } \\
\hline Single & 84.4 & 94.6 & 94.4 & 88.7 \\
\hline Married & 15.6 & 5.4 & 5.6 & 11.3 \\
\hline $\mathrm{X}^{2}(\mathrm{P}-$ value $)$ & \multicolumn{2}{|c|}{$3.398(.065)^{\mathrm{NS}}$} & \multicolumn{2}{|c|}{$6.960(.008)^{* *}$} \\
\hline \multicolumn{5}{|l|}{ Religion } \\
\hline Muslim & 78.1 & 78.5 & 76.1 & 81.1 \\
\hline Cristian & 21.9 & 21.5 & 23.9 & 18.9 \\
\hline $\mathrm{X}^{2}(\mathrm{P}-$ value $)$ & \multicolumn{2}{|c|}{$1.002(.572)^{\mathrm{NS}}$} & \multicolumn{2}{|c|}{$1.597(.206)^{\mathrm{NS}}$} \\
\hline \multicolumn{5}{|l|}{ Mothers' education } \\
\hline Illiterate & 31.3 & 34.4 & 29.6 & 39.6 \\
\hline Primary or Preparatory & 25.0 & 28.0 & 26.8 & 26.4 \\
\hline Secondary & 28.1 & 25.8 & 33.8 & 17.0 \\
\hline University & 15.6 & 10.8 & 8.5 & 17.0 \\
\hline $\mathrm{X}^{2}(\mathrm{P}-$ value $)$ & \multicolumn{2}{|c|}{$2.660(.082)^{\mathrm{NS}}$} & \multicolumn{2}{|c|}{$6.089(.010)^{*}$} \\
\hline
\end{tabular}

$N S=$ Not statistically significance $\quad *$ Statistically significant at $P-$ value $\leq 0.05 \quad * *$ Statistically significant at $P-$ value $\leq .01$ 
Table (9): Correlation between studied student's knowledge, beliefs and attitudes about oocyte cryopreservation in pretest and posttest, $(n=125)$.

\begin{tabular}{|c|c|c|c|c|c|c|}
\hline \multirow{2}{*}{ Variables } & \multicolumn{3}{|c|}{ Pretest } & \multicolumn{3}{|c|}{ Posttest } \\
\hline & Knowledge & Beliefs & Attitudes & Knowledge & Beliefs & Attitudes \\
\hline \multicolumn{7}{|l|}{ Knowledge } \\
\hline r. value & 1 & .111 & .406 & 1 & .172 & .227 \\
\hline P. value & - & .108 & $.022 *$ & - & $.020 *$ & $.006 * *$ \\
\hline \multicolumn{7}{|l|}{ Beliefs } \\
\hline r. value & .111 & 1 & .270 & .172 & 1 & .469 \\
\hline P. value & .108 & - & $.050 *$ & $.020 *$ & - & $.007 * *$ \\
\hline \multicolumn{7}{|l|}{ Attitudes } \\
\hline r. value & .406 & .270 & 1 & .227 & .469 & 1 \\
\hline P. value & $.022 *$ & $.050 *$ & - & $.006 * *$ & $.007 * *$ & - \\
\hline
\end{tabular}

Table (1): Shows that $60.8 \%$ of the age of studied students was ranged between $18<20$ yrs. with mean age $19.35 \pm 1.2$ yrs., $92.0 \%$ of them were single, 78.4 $\%$ were Muslim, $73.6 \%$ were come from rural area and $82.4 \%$ of them had moderate social class. Concerning to their mother's education, $34.4 \%$ of their mothers were illiterate.

Table (2): Regarding to definition of egg freezing, $20.0 \%$ of studied students answered completely correct about the meaning of oocyte cryopreservation in pretest, increased to $52.0 \%$ in posttest.

Concerning to indications for oocyte cryopreservation, $24.8 \%$ of them answered completely correct in pretest, increased to $76.0 \%$ in posttest. Relating to question "There is a significant decrease in a woman's fertility with advanced age?" $50.4 \%$ of them answered correctly in pretest increased to $92.0 \%$ in posttest. As regard to "oocyte cryopreservation can prolong a woman's fertility before the age of 35 yrs.," $36.8 \%$ of them answered correctly in pretest increased to $72.0 \%$ in posttest.

As regard to "eggs numbers that should be stored to attain conception later on, $16.8 \%$ of them answered correctly in pretest, $61.6 \%$ of them answered " $20-30$ egg." in posttest. Concerning to barriers for oocyte cryopreservation, only $2.4 \%$ of them answered completely correct in pretest, increased to $15.2 \%$ in posttest.

Figure (1): Regarding to sources of information about oocyte cryopreservation, the main sources were social media $(46.4 \%)$ followed by school and university $(25.6 \%)$ and physician and nurses $(11.2 \%)$

Table (3): Illustrates that $14.4 \%$ and $60.0 \%$ of studied students were believed that "women should preserve her fertility through oocyte cryopreservation." in pretest and posttest respectively. Concerning to cost of oocyte cryopreservation, $6.4 \%$ and $28.0 \%$ of them were believed that "the cost should be covered by national health care system" in pretest and posttest respectively, also $33.6 \%$ and $16.0 \%$ of them were supposed that " the high cost of oocyte cryopreservation is a barrier for it" in pretest and posttest respectively. Furthermore $17.6 .0 \%$ and $49.6 \%$ of them were thought that "oocyte cryopreservation should be a part of premarital counselling." in pretest and posttest respectively.

Table (4): Illustrates that $55.2 \%$ and $72.0 \%$ of studied students were agree with "egg preservation should be encouraged" in pretest and posttest respectively. Concerning to application of egg preservation in Egypt, $44.8 \%$ and $28.0 \%$ of them were believed that "we do not apply egg preservation in Egypt" in pretest and posttest respectively. Furthermore $64.0 \%$ and $24.8 \%$ of them were thought that "egg preservation is very expensive and cannot be feasible to anyone" in pretest and posttest respectively. Additionally, $30.4 \%$ and $68.0 \%$ of them were agree with "Oocyte freezing may be a possible option for you" in pretest and posttest respectively.

Table (5): Demonstrates that $55.2 \%$ of studied students had poor knowledge about oocyte cryopreservation in pretest reduced to $17.6 \%$ of them in posttest with highly statistically significant improvements in their knowledge level. Furthermore $60.0 \%$ of them had negative beliefs about egg freezing in pretest vs $33.6 \%$ of them in posttest with statistically significant improvements in their beliefs. Additionally, only $25.6 \%$ of them had positive attitude about egg freezing in pretest increased to $56.8 \%$ in posttest with statistically significant improvements in their attitudes.

Table (6) : Shows that there were highly statistical significant relation between students' total knowledge about oocyte cryopreservation and their demographic data related to their age, academic level, marital status and their mothers education in both pretest and posttest where P-value were $0.030,0.000,0.002$ and 
0.003 respectively in pretest and were $0.033,0.001$, 0.003 and 0.002 respectively in posttest.

Table (7): Shows that there were statistical significant relation between students' total beliefs about oocyte cryopreservation and their age, academic level and their socioeconomic level in pretest where P-value were $0.050,0.053$ and 0.000 respectively, while there were statistical significant relation between students' total beliefs about egg freezing and their academic level and marital status only in posttest where P-value were 0.049 and 0.051 respectively.

Table (8): Shows that there were statistical significant relation between students' total attitudes toward oocyte cryopreservation and their age, academic level and residence in pretest where $\mathrm{P}$-value were $0.008,0.002$ and 0.034 respectively, while there were statistical significant relation between students' total attitudes and their age, socioeconomic level, marital status and their mothers education in posttest where P-value were $0.007,0.032,0.008$ and 0.010 respectively.

Table (9): Reveals that there was moderate positive correlation between knowledge and attitudes about oocyte cryopreservation of studied students and weak positive correlation between their beliefs and attitudes about oocyte cryopreservation in pretest where $r_{-}$ value was .406 and .270 respectively with statistically significant differences where $p_{\text {_ }}$ value was 0.022 and 0.050 respectively.

Additionally, there were moderate positive correlations between knowledge, beliefs and attitudes about oocyte cryopreservation of studied students after implementation of the educational guidelines where $r_{-}$value was $0.172,0.227$ and 0.469 respectively with statistically significant differences where $p_{-}$value was $0.020,0.006$ and 0.007 respectively.

\section{Discussion}

Cryopreservation of oocytes has been labeled as a 'revolution for women's release and reproductive autonomy. Motherhood can be postponed for a variety of reasons, including a desire to focus on their job, the waiting for a suitable spouse or the belief that they are not 'ready'. It has the potential to provide woman more reproductive choices; including the opportunity to choose when and with whom they want to have children, (Argyle et al., 2016).

The current study findings showed that about two thirds of students' age ranging from $18<20$ years and majority of them were single. It was in the same line with the study conducted by (Fotopoulou et al., (2015) in Athens, which found that, students' mean age was twenty yrs. and Tozzo et al., (2019) this study carried out in Italy and found that the majority of participants, were between eighteen and twentytwo years old and half of them were single.

Regarding definition of egg freezing, one fifth of studied students answered correct about the meaning of oocyte cryopreservation in pretest. It disagree with the findings of Tozzo et al., (2019) who showed that the majority of nursing students do not understand what egg freezing means.

Concerning to indications for oocyte cryopreservation, about one third of them answered completely correct that women who wants to postpone pregnancy, diagnosed with cancer, with a family history of early menopause and grand nulliparous) were the main group who seek this service. It was similar to study conducted by Argyle et al., (2016) which showed that egg freezing is an essential fertility preferences for women with more than a few medical conditions a side from cancer as instance, women with endometriosis who may suffer from reduced ovarian reserve post-surgery, women with autoimmune illness requiring gonadotoxic treatment and women with hereditary anomalies leading to reduced fertility or risk of early menopause. It also was contradicted by study conducted by Mahesan et al., (2019) in Eastern Virginia which found that two thirds of students overall would consider egg freezing for career reasons, less than half of them would reflect it for medical reasons, and only one third would reflect it for social reasons.

More than half of students were aware that woman's fertility is decreased significantly with advanced age. It was supported by the findings of study conducted by Argyle et al., (2016) which discovered that fertility decline and accelerates after the age of 35. This deterioration is caused by a decrease in the number of follicles and the quality of oocyte, as well, it was in similarity with Ikhena, et al., (2017) which found that three quarters of respondents believed that at the age of $35 \mathrm{yrs}$. women fertility decreased significantly, and study carried out by Meissner et al., (2016) in Hannover, Germany, which founded that nearly half of students were knowledgeable that a first decrease in reproductivity of women starts before the age of 30 years and about one third of the students incorrectly believed that there is an obvious decline of women's fertility after the 40 yrs. Additionally, it was in agreement with Fotopoulou et al., (2015) which found that majority of participants thought that the reproductivity of women begin to weakening after $35 \mathrm{yrs}$. of age.

Relating to "the age at which a woman can effectively freeze her oocytes", more than half of them don't know the answer in pretest, while about 
two thirds of them answered correctly "25-30 yrs." in posttest. From researchers' points of view, it may be justified as the educational guidelines were effective on improving their knowledge related to this question. It was supported by the findings of study conducted by Tozzo et al., (2019) which revealed most respondents believed that at 26-30 yrs. of age, they may experiencing egg freezing, also Will et al., (2017) found that more than half of respondents answered that a woman can effectively freeze her oocytes at 25-29 yrs. of age. Additionally, Ikhena, et al., (2017) revealed that the highest percentage of participants selected 25-28 yrs., and one third of them selected 30-33 yrs.

As regard to the eggs numbers that should be stored to attain conception later on?", more than two thirds of them answered "Don't know" in pretest, while about two thirds of them answered "20-30 egg." in posttest. It was in similarity with the findings of Mahesan et al., (2019) which stated that, for age 40 , one third of participants chosen 21-30 eggs, and one quarter of them selected 40 eggs.

The findings of the present study illustrated that, about one third of participants don't know these barriers in pretest. From researchers point of views, this may be due to that above half of studied students have no previous knowledge about oocyte cryopreservation and don't have a clear idea about barriers of it. Also, a vast minority of them answered correctly that (the high cost, social stigma, ethical or moral and religious concerns, egg may be changed with another women, no support from family and husbands) were barriers of oocyte cryopreservation in pretest, increased to less than one fifth in posttest that may be due to effectiveness of the educational intervention on their knowledge.

Additionally, the main barriers for oocyte cryopreservation in posttest were the high cost of the procedure followed by social stigma. It was coming in agreement with Will et al., (2017) who found that the most frequently barriers were finances and time commitment, then being uncertain of available decisions, potential health implications, and social stigma.

The main sources of students' information about oocyte cryopreservation were social media followed by school or university, physician or nurses, friends and mothers. This can be justified that this age group of youth is interested with social media and stay long time using it and social media is always easily accessible for them providing them with a variety of information in different fields. Friends and mothers were the least sources of information as above one third of their mothers were illiterate besides, they may be known little about egg preservation and their friends may be have no or little knowledge about it.
It was supported by the study of Fotopoulou et al., (2015), which revealed that the main sources of information were internet, journals or/and TV followed by university, family members, health professionals and friends. As well, previous findings were contradicted with Tozzo et al., (2019) which revealed that more than one third of the participants have heard about oocyte cryopreservation from their family, friends and media and school or university. Also, Will et al., (2017) showed that the most frequently source was the formal education, followed by social media and friends.

In addition, almost half of participants had poor knowledge about oocyte cryopreservation in pretest reduced to less than one fifth of them in posttest with highly statistically significant improvements in their knowledge level. Furthermore, about two thirds of them had negative beliefs about egg freezing in pretest vs one third of them in posttest with statistically significant positive changes in their beliefs.

Additionally, only one quarter of them had positive attitude about egg freezing in pretest increased to more than half of them in posttest with statistically significant improvements in their attitudes. From researchers' point of view, it may be due to effectiveness of the educational guidelines that given to students and it had a greater impact on changing students' knowledge, attitudes and beliefs about egg freezing. These findings were supported by study carried out by (Will et al., 2017) which revealed that the mean knowledge score of pre-tests and the percentage of correct answers after the intervention increased significantly. Also, agreed with a study conducted by (Rafiei et al., 2020), which found that a significant difference in mean score of participants' knowledges was founded pre and post the educational intervention.

Regarding the Relation between studied student's knowledge about oocyte cryopreservation and their socio-demographic characteristics, a highly statistical significant relation between students' total knowledge about oocyte cryopreservation and their age, academic level, marital status and their mother's education were founded in both pretest and posttest. This doesn't match the study of Rafiei et al., (2020) who revealed that knowledge score is not significantly different between different sex, residence and marital status post education program.

Moreover, statistically significant relation was founded between students' total attitudes toward oocyte cryopreservation and their age, academic level and residence in pretest, while there was statistically significant relation between students' total attitudes and their age, socioeconomic level, marital status and their mother's education in posttest. This partially in 
agreement with Mahesan et al. (2019) who found that, at age of 31-35yrs, participants preferred experiencing egg freezing while highest percentage of undergraduate would carry it at age of 26-30 yrs.

By looking to Correlation between studied student's knowledge, beliefs and attitudes about oocyte cryopreservation in pretest and posttest, it was found that there was moderate positive correlation between knowledge and attitudes about oocyte cryopreservation of studied students and weak positive correlation between their beliefs and attitudes about oocyte cryopreservation in pretest with statistically significant differences.

Additionally, after implementation of the educational guidelines statistically a significant differences with moderate positive correlations between knowledge, beliefs and attitudes about oocyte cryopreservation of studied students. This came in contact with (Farrag, et al, 2020) whose study revealed that there was a statistically significant relation between the knowledge of couples and their perception. These findings were in harmony with (Rashed, et al., 2018) who found that, there was a significant, positive relation between knowledge and attitude before and after counseling ( $\mathrm{r}=0.49, \mathrm{r}=0.78$ respectively). This may rendered to improving the students' knowledge regarding oocyte cryopreservation can positively reflected on their attitude and beliefs because increasing awareness can modify their misconceptions and clarify any ambiguities about the procedure and also came in contact with the new technologies and make benefit of it.

According to what is discussed above, we can make use of the findings of the current study, not only through increasing awareness of nursing students about oocyte cryopreservation but also changing their beliefs, perception and attitudes toward it. They should be alert about fertility preservation choices. It is their primary responsibilities to communicate with couples in different community setting including Maternal and Child Health centers (MCH) through premarital counselling, infertility clinics, In-Vitro fertilization centers and hospitals. They can disseminate the acquired knowledge to the whole community as they have more relations with patients and their families if compared with other healthcare professionals, they help couples to modify their attitudes and change their behaviors that will guide them to take the right decision about cryopreservation process.

\section{Conclusion:}

According to the results of the current study we can concluded that there were highly statistically significant differences in students' knowledge level about oocyte cryopreservation after implementation of educational guidelines. It reflects the greater impact of provided educational guidelines on changing students' knowledge, attitudes and beliefs about oocyte cryopreservation.

Also, There were moderate positive correlations between knowledge, beliefs and attitudes about oocyte cryopreservation of studied students after implementation of the educational guidelines.

\section{Recommendations: -}

- According to our results, more efforts needed by the health care system, educational institutions, and all public media to reduce the gab in fertility awareness between the youth.

- There are a need for supporting and improvement of facilities related to introduce service and economic support for fertility preservation.

- Provide a continuous planned educational courses on regular basis for different non-medical faculties to improve their knowledge, beliefs and attitudes about oocyte cryopreservation.

- Further studies involving the partners during premarital counseling and family members in different maternal and child health centers $(\mathrm{MCH})$ to increase their awareness about oocyte cryopreservation to improve their quality of life later on and to remove barriers related to them.

\section{References}

- Abdelwahab S., \& Samy M. (2017): Obstacles facing oocyte cryopreservation in the Middle East. Obstetrics \& Gynecology International Journal, 7(2), 00238.

- Argyle C., Harper J., \& Davies, M. (2016): Oocyte cryopreservation: where are we now? Human reproduction update, 22(4), 440-449.

- Biswas G., \& Selvaraj P. (2018): Legal and Ethical Issues in Oocyte Cryopreservation. Recent Advances in Forensic Medicine and Toxicology-2: Good Practice Guidelines and Current Medicolegal Issues, 2, 61 .

- Crawford N., \& Steiner A. (2015): Age-related infertility. Obstetrics and Gynecology Clinics, 42(1), 15-25.

- Daniluk J., \& Koert E. (2016): Childless women's beliefs and knowledge about oocyte freezing for social and medical reasons. Human reproduction, 31(10), 2313-2320.

- European Society of Human Embryology and Reproduction (ESHER) (2016): Annual Scientific Meeting Lisbon, Portugal 14-17 June.

- Fotopoulou V., Chasiakou A., Gryparis A., \& Baka S. (2015): Greek medical student's knowledge and attitudes towards infertility and 
assisted reproductive technologies (Greek medical students and ART). Women's Health Care, 4, 4-7.

- Ghraib, S., \& Khait, A., (2017): The Relationship between Primary Infertility and Depression among Women Attending Royal Medical Services Hospitals in Jordan. J Community Med Health Educ, 7, 2161-0711.1000533.

- Gordan B. \& Kuttner, (2015): Article on Delayed Childbearing Trends, FERTILITYTODAY.ORG,http://www.fertilitytoda y.org/age_infertility. tm (follow "Read an Article on Delayed Childbearing Trends by Gordan Kuttner, MD" hyperlink) (last visited Jan. 25, 2015).

- Ikhena-Abel D., Confino R., Shah N., Lawson A., Klock S., Robins J., \& Pavone M. (2017): Is employer coverage of elective egg freezing coercive?: a survey of medical students' knowledge, intentions, and attitudes towards elective egg freezing and employer coverage. Journal of assisted reproduction and genetics, 34(8), 1035-1041.

- Inhorn M., Birenbaum-Carmeli D., Birger J., Westphal L., Doyle J., Gleicher N., \& Patrizio P. (2018a): Elective egg freezing and its underlying socio-demography: a binational analysis with global implications. Reproductive Biology and Endocrinology, 16(1), 1-11.

- Inhorn M., Birenbaum-Carmeli D., Westphal L., Doyle J., Gleicher N., Meirow D., \& Patrizio P. (2018b): Ten pathways to elective egg freezing: a binational analysis. Journal of assisted reproduction and genetics, 35(11), 2003-2011.J.C.

- Kim R., Yoon T.K., Kang I.S., Koong M.K., Kim Y.S. \& Kim M.J., (2018): Decision making processes of women who seek elective oocyte cryopreservation. J Assist Reprod Genet ;35:162330.

- Lallemant C., Vassard D., Nyboe Andersen A., Schmidt, L., \& Macklon, N. (2016): Medical and social egg freezing: internet-based survey of knowledge and attitudes among women in Denmark and the UK. Acta obstetricia et gynecologica Scandinavica, 95(12), 1402-1410.

- Mahesan A., Sadek S., Ramadan H., Bocca S., Paul A., \& Stadtmauer L. (2019): Knowledge and attitudes regarding elective oocyte cryopreservation in undergraduate and medical students. Fertility research and practice, 5(1), 1-7.

- Meissner C., Schippert C., \& Von VersenHöynck F. (2016): Awareness, knowledge, and perceptions of infertility, fertility assessment, and assisted reproductive technologies in the era of oocyte freezing among female and male university students. Journal of Assisted Reproduction and Genetics, 33(6), 719-729.
- Nasab S., Ulin L., Nkele C., Shah J., Abdallah M. E., \& Sibai B. (2020): Elective egg freezing: what is the vision of women around the globe?. Future Science OA, 6(5), FSO468.

- Nicolette A. (2016): Empty benefits: employersponsored oocyte cryopreservation and potential for employment discrimination. Hastings Women's LJ, 27, 341 .

- Rafiei N., Khosravizadeh Z., Zanjani S., \& Khodamoradi K. (2020): 315 The Impact of Educational Package on Nursing Students' Knowledge Towards Fertility Preservation, A Quasi-experimental Study. The Journal of Sexual Medicine, 17(1), S77.

- Farrag R., \& El-tohamy, N. (2020): Oocyte Cryopreservation: Awareness and Perception of Infertile Couple Undergoing In-Vitro Fertilization. Egyptian Journal of Health Care, 11(4), 300-321.

- Rashed A., Ismaeel N. \& Kamel M., (2018): Cryopreservation Counseling and Its Effect on Knowledge and Attitude of Young Female Cancer Patients. Journal of Health, Medicine and Nursing, Vol.54. 747-755.

- Rodriguez-Wallberg K., Hao X., Marklund A., Johansen G., Borgström B., \& Lundberg F. (2021): Hot Topics on Fertility Preservation for Women and Girls-Current Research, Knowledge Gaps, and Future Possibilities. Journal of Clinical Medicine, 10(8), 1650.

- Stoop D., Cobo A., \& Silber S. (2014): Fertility preservation for age-related fertility decline. The Lancet, 384(9950), 1311-1319.

- Tozzo P., Fassina A., Nespeca P., Spigarolo G., \& Caenazzo L. (2019): Understanding social oocyte freezing in Italy: a scoping survey on university female students' awareness and attitudes. Life sciences, society and policy, 15(1), 1-14.

- Will E., Maslow B., Kaye L., \& Nulsen J. (2017): Increasing awareness of age-related fertility and elective fertility preservation among medical students and house staff: a pre-and post-intervention analysis. Fertility and sterility, 107(5), 1200-1205. 